\title{
La Caliza de Sierra Elvira: comportamiento petrofísico de una piedra significativa del Patrimonio Arquitectónico Andaluz
}

\section{The Sierra Elvira limestone: petrophysical features of a widespread Andalusian decorative material}

\author{
E. Sebastián Pardo $(*)$, G. Cultrone ${ }^{(*)}$, V. Garibaldi(**), C. Rodríguez Navarro(*), \\ M. J. de la Torre ${ }^{(* * *)}$ y I. Valverde ${ }^{(* * * *)}$
}

Recepción/Received: 15-V-07

Aceptación/Accepted: 09-X-07

Publicado online/Online publishing: 19-XII-07

\section{RESUMEN}

La "Piedra de Sierra Elvira" constituye una de las piedras ornamentales más significativas del Patrimonio Arquitectónico de Andalucía Oriental. Es una roca caliza del Lias que aflora en el Subbético Medio del sector central de las Cordilleras Béticas. El litotipo más explotado es una caliza con crinoides, en bastante menor importancia se ha extraído también otra caliza micrítica de la misma edad. Los bancos son muy potentes, en algunos casos de más de $5 \mathrm{~m}$, con un paralelismo constante y un buzamiento que favorece su explotación en los frentes de cantera.

Sus características petrográficas y físico-mecánicas le confieren una calidad técnica y durabilidad muy notables para su utilización como material de construcción estructural y ornamental. Los principales parámetros que determinan estas cualidades son su mínima porosidad eficaz y asociado a ello sus excelentes propiedades hídricas, así como sus elevadas resistencias mecánicas y el bajo coeficiente de anisotropía textural, tanto elástico como mecánico. Este conjunto de características ha permitido su utilización en muy diversas funciones constructivas.

Palabras clave: caliza, piedra natural, parámetros físico-mecánicos, durabilidad, Patrimonio Arquitectónico.

\section{SUMMARY}

"Sierra Elvira stone" is one of the ornamental building stones most widely used in the historical monuments of eastern Andalusia. A Liassic age limestone, it appears in the central section of the Baetic Mountains and more specifically in the Middle Subbaetic domain. While the most common variety is a crinoid limestone, a micritic limestone of the same age has also been quarried, albeit in much smaller quantities. These stones form very thick beds, up to nearly $5 \mathrm{~m}$ deep, that run in consistently parallel lines and have a dip angle that facilitates quarrying.

With petrographic, physical and mechanical properties that ensure stone strength and durability, it is a high quality building material suitable for both structural and ornamental purposes. These properties can be attributed to the minimal open porosity and concomitant excellent water resistance that characterize the stone, as well as to its high mechanical strength and low textural anisotropy, both elastic and mechanical. With such attributes, the stone can be successfully used for any number of construction purposes.

Keywords: limestone, natural stone, physical and mechanical properties, durability, Architectural Heritage.

(*) Universidad de Granada (Granada, España).

(**) Fondazione Cariplo. Area Ambiente (Milano, Italia).

$(* * *)$ Universidad de Jaén (Linares, España).

$(* * * *)$ Escuela Universitaria de Arquitectura Técnica de Granada (Granada, España). 


\section{INTRODUCCIÓN}

La piedra de Sierra Elvira ha desempeñado, y aún lo hace, un importante papel en el sector de la construcción de Andalucía Oriental y especialmente en la provincia de Granada. Su empleo en las distintas épocas ha tenido una mayor o menor relevancia en función de los criterios culturales y artísticos que en ese momento dominaran.

En el sector industrial de la Piedra Natural, la Piedra de Sierra Elvira se conoce como Bronceado Gris Sierra Elvira (1) por su diversidad de tonos cromáticos que varían desde el gris oscuro al color bronce verdoso. Resulta llamativo como para muchos historiadores este tipo de piedra constituye el denominado "mármol verde de Sierra Elvira" $(2,3)$.

Del amplio conjunto de materiales rocosos que afloran en este ámbito geográfico, prácticamente la totalidad de carácter calcáreo, los más utilizados como rocas ornamentales han sido las calizas de crinoides y de forma más minoritaria otra variedad de calizas de textura marcadamente micrítica. Esta última se conoce también como "piedra losa"; su extracción prácticamente cesó a finales de la década de los años treinta.

La caliza de crinoides (equinodermos fósiles cuyo tronco está formado por segmentos de morfologías variadas: pentagonales, estrelladas, circulares, etc.) se ha utilizado tanto en la arquitectura religiosa como en la arquitectura civil y residencial (4). Se encuentra en portadas, fuentes, estatuas, columnas, zócalos de edificios, solerías, bordillos de plazas o calles, etc. Algunos de los edificios históricos más significativos de Granada en los que se utilizó esta piedra ornamental son: el Hospital Real, el Palacio de Carlos V en la Alhambra, la Real Chancillería, la Catedral, las iglesias de nuestra Señora de las Angustias, San Juan de Dios, San Justo y Pastor (Figura 1).

\section{ESTRATIGRAFÍA Y CANTERAS}

Los relieves de Sierra Elvira se consideran, en términos geológicos un horst de materiales de edad mesozoicos, que se elevaron hasta los 1100 m respecto a la depresión de Granada (500-600 m). Representan la parte más meridional del Subbético Medio en el sector central de las Cordilleras Béticas; están constituidos por materiales triásicos, jurásicos y cretácicos, cuyo origen se relaciona con el desarrollo del mar del Thetys.

Las calizas de crinoides y las micríticas del Lias (Carixiense Inferior-Medio) de la secuencia estratigráfica de Sierra Elvira $(5,6)$ constituyen el último término que se depositó antes de que se rompiera la gran plataforma carbonatada del borde meridional de la placa europea

\section{INTRODUCTION}

Throughout history and even today, Sierra Elvira stone has played an important role in the building industry in eastern Andalusia and in particular in the province of Granada. Its popularity has varied in different historical periods depending on contemporary cultural and artistic preference.

In the natural stone industry, Sierra Elvira stone is known as Bronceado Gris Sierra Elvira (bronze grey Sierra Elvira) (1) because of the diversity of its coloration, which ranges from dark grey to greenish bronze. Tellingly, many historians refer to this type of stone as "Sierra Elvira green marble" $(2,3)$.

Almost all the many varieties of rock outcropping in this area are calcareous, and the one most widely used for ornamental purposes is crinoid limestone. A second variety with a markedly micritic texture known as "slab stone", also used in the past although to a lesser extent, has since fallen out of favour and has barely been quarried since the nineteen thirties.

Crinoid limestone (containing fossil echinoderms, organisms whose segmented trunks adopted a variety of shapes: pentagonal, stellar, circular and so forth.) has been used in churches as well as in civil and residential buildings (4). It can be seen on facades, fountains, columns and plinths in some of Granada's most emblematic historic buildings, including the Royal Hospital, the Palace of Charles $V$ on the grounds of the Alhambra, the Royal Chancellery, the Cathedral and Our Lady of Sorrows, St John of God and Saints Justus and Pastor Churches (Figure 1).

\section{STRATIGRAPHY AND QUARRIES}

Geologically speaking, Sierra Elvira is a Mesozoic horst that rises from the Granada depression (500-600 m) to an elevation of $1100 \mathrm{~m}$, and comprises the southernmost part of the Middle Subbaetic domain in the central section of the Baetic Mountains. Its Triassic, Jurassic and Cretaceous materials were deposited on the Tethys seabed.

The Liassic age (Early-Middle Carixian) crinoid and micritic limestone from the Sierra Elvira stratigraphic sequence (5, 6) was the last geological formation to be deposited before the great carbonate platform on the southern rim of the 


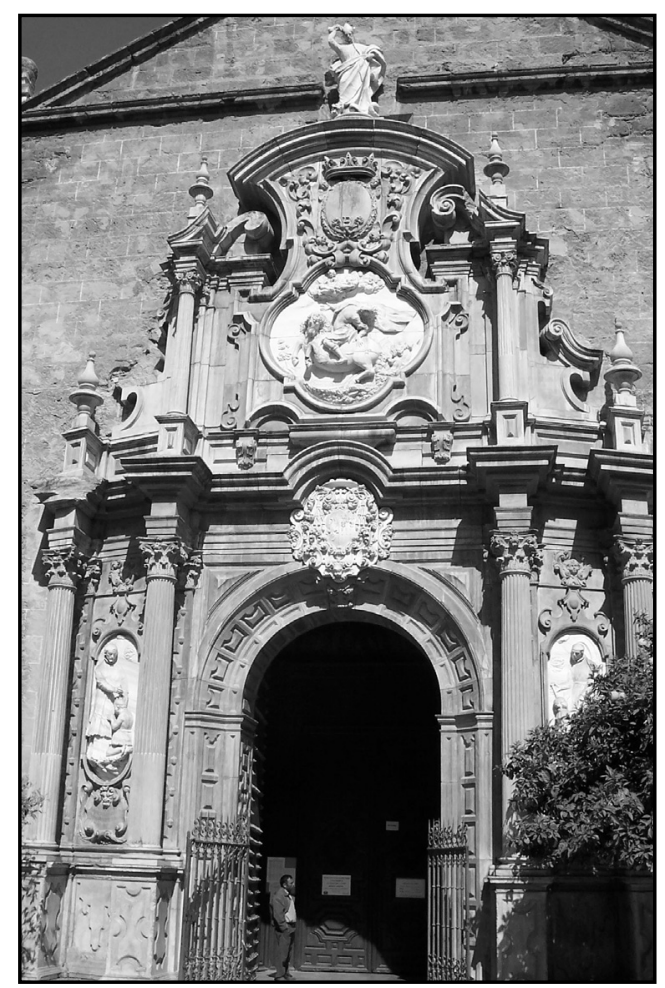

Figura 1. Portada de la iglesia de San Justo y Pastor (Granada). Constituida por caliza de crinoides y mármol blanco.

Figure 1. Crinoid limestone and white marble portal on Saints Justus and Pastor Church, Granada.

(ruptura que se verificó alrededor de los 180 M.A. como consecuencia de la apertura del Océano Atlántico).

Estratigráficamente se diferencian dos conjuntos; el inferior (unos $20 \mathrm{~m}$ de potencia) se presenta como una gran bancada de calizas con laminación unidireccional a gran escala y con una elevada densidad de estilolitos. La potencia de los estratos, en algunos casos, supera los cinco metros (Figura 2). El superior se caracteriza por tener laminación cruzada del tipo hardground asociado a grietas poligonales de desecación, potencia alrededor de $5 \mathrm{~m}$. La microfauna está constituida, además de los crinoides, por fragmentos de moluscos, braquiópodos y foraminíferos.

La extracción de piedra ornamental en España representa uno de los sectores económicos más importantes y dinámico; en la provincia de Granada esta actividad encuentra sus raíces ya en época romana. Así, en el Museo Arqueológico de la ciudad se conserva una lápida funeraria de ese periodo, elaborada con caliza de crinoides de Sierra Elvira. En algunas canteras antiguas aún se perciben las huellas de extracción de varios fustes de columnas dejados en la roca por canteros árabes.

El patrimonio lapídeo de Sierra Elvira se ha explotado, sobre todo en el caso de la caliza de crinoides, en forma de bloques regulares de grandes dimensiones con fines
European plate broke with the formation of the Atlantic Ocean around 180 million years ago.

Two stratigraphic levels can be clearly distinguished. The lower and thicker (about $20 \mathrm{~m}$ ) of the two is characterized by large-scale unidirectional lamination and a high density of stylolites. Some of the beds in this level are up to five metres thick (Figure 2). The upper level (about 5 $m$ ) is characterized by hardground-like cross-lamination associated with polygonal cracks caused by desiccation. In addition to crinoids, other microfauna fossils are present, including fragments of molluscs, brachiopods and foraminifers.

Ornamental stone quarrying has always been one of Spain's most important and dynamic industries. In the province of Granada it can be traced back to Roman times: indeed, the city's Archaeological Museum exhibits a Roman gravestone made with Sierra Elvira crinoid limestone, while the column shaft excavation marks left by Arab quarrymen are still visible in some old quarries.

At Sierra Elvira, crinoid limestone was normally quarried in large, even blocks for ornamental use, while other kinds of limestone and dolomite were mainly used for 


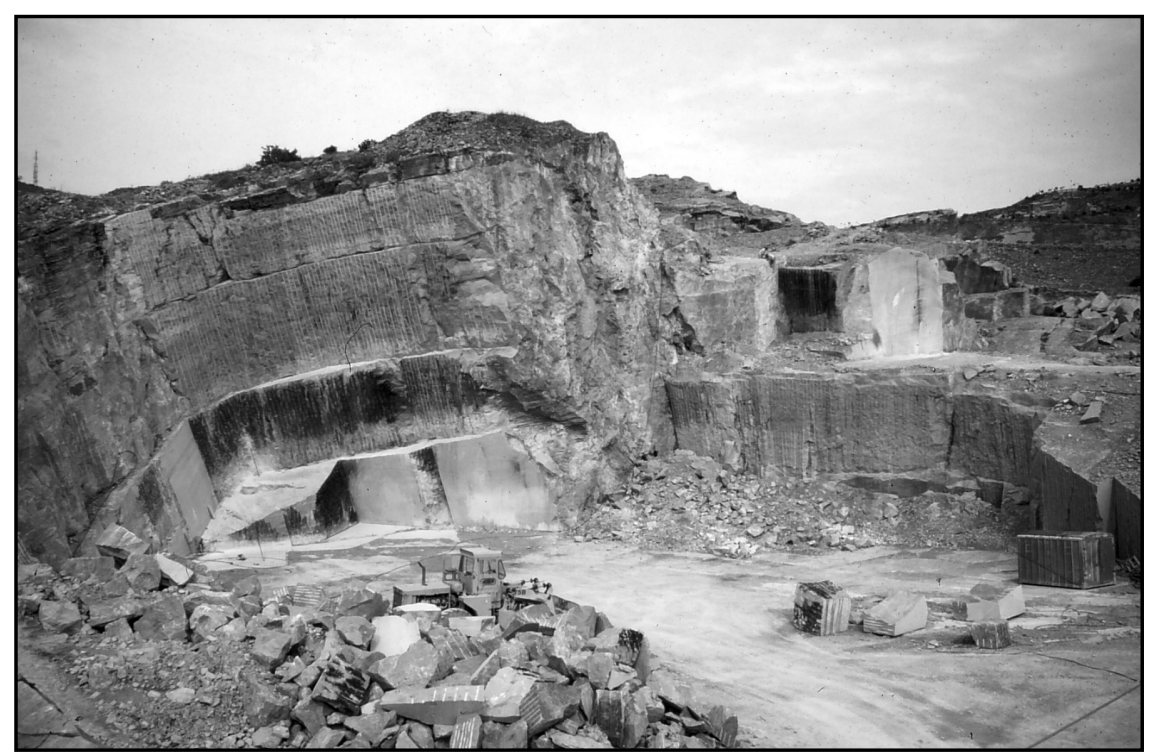

Figura 2. Cantera de caliza de crinoides "Ermita Tres Juanes" (Atarfe, Granada).

Figure 2. "Ermita Tres Juanes" crinoid limestone quarry (Atarfe, Granada).

ornamentales, mientras que otros tipos de calizas y dolomías, se han extraído fundamentalmente para áridos. El distrito de canteras se extiende entre los municipios de Atarfe, Pinos Puente y Albolote, aunque la mayor parte de las canteras de caliza de crinoides se encuentran en el término de Atarfe (Figura 3).

La extracción realizada mediante voladura controlada resulta favorecida por el ángulo de buzamiento (por tér- aggregate. Quarries were scattered across the area delimited by the towns of Atarfe, Pinos Puente and Albolote, although most of the crinoid limestone was quarried within the municipal district of Atarfe (Figure 3).

Controlled blasting is facilitated by the dip angle (an average $30^{\circ}$ ), consistently parallel beds, presence of planes

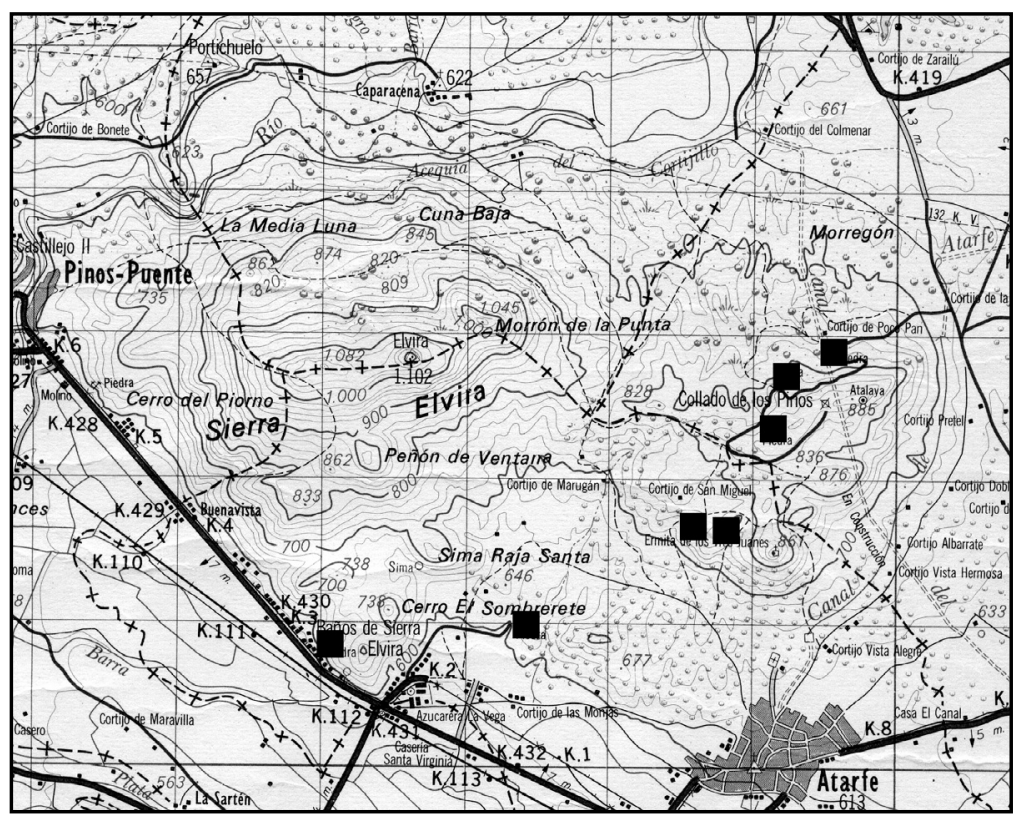

Figura 3. Mapa topográfico de las canteras (cuadrados negros) en Sierra Elvira (Atarfe, Granada).

Figure 3. Map of the Sierra Elvira quarries (black squares) at Atarfe, Granada. 
mino medio unos $30^{\circ}$ ) y el paralelismo constante de los estratos; asimismo, por la presencia de planos de debilidad mecánica y niveles muy finos de sedimentos limosos paralelos o subparalelos a la estratificación.

La zona extractiva actualmente está declarada Parque Natural Periurbano con la consiguiente normativa de protección, lo que condiciona fuertemente la explotación de este material pétreo.

\section{MATERIALES Y MÉTODOS}

Procedentes de los relieves de Sierra Elvira han sido proporcionados por las canteras "Los Órganos 2" y "La Sombra" de Atarfe (Granada) bloques de calizas de crinoides y micríticas. El estudio mineralógico y textural se ha efectuado mediante difracción de rayos $X(D R X)$, método de polvo desorientado, y microscopía óptica con luz polarizada. En el primer caso se ha usado un difractómetro Philips PW1710, equipado con rendija automática, y en el segundo un microscopio petrográfico Olympus BX-60.

Para establecer el color de las calizas se ha utilizado un espectrofotómetro HunterLab MiniScan 4000S cuya fuente de luz es una lámpara de tungsteno. Se han obtenido los valores triestimulares $(X, Y, Z)$, de luminosidad $\left(L^{*}\right)$, cromaticidad $\left(\mathrm{a}^{*}, \mathrm{~b}^{*}\right)$, color $\left(\mathrm{C}^{*}\right)$ y ángulo de matiz $\left(\mathrm{H}^{*}\right)$.

Los datos de porosidad de las dos variedades de caliza se han determinado mediante porosimetría de inyección de mercurio (PIM), utilizando un equipo Micromeritics AutoPore III modelo 9410, y ensayos hídricos, llevando a cabo pruebas de absorción libre (7) y desorción (8) sobre probetas cúbicas de $5 \mathrm{~cm}$ de arista.

Las medidas de ultrasonidos han permitido calcular la velocidad de las ondas elásticas longitudinales VP en las dos variedades de caliza. Se ha utilizado un generador de pulsos ultrasónicos marca Controls, modelo E46/3 con transductores de $54 \mathrm{kHz}$.

Finalmente, mediante los ensayos mecánicos, se ha determinado la resistencia a la deformación (longitudinal y trasversal), compresión, tracción y flexión. En el primer caso se ha utilizado un extensímetro eléctrico Hottinger HBM 20/120 con muestras que medían $7 \times 7 \times 21 \mathrm{~cm}$. Para el ensayo de compresión uniaxial se ha utilizado una prensa Metro Com MI 30 con una capacidad de carga de $3 \times 10^{5} \mathrm{~kg}$. El ensayo se ha realizado en seco sobre probetas cúbicas de $7 \mathrm{~cm}$ de arista. La resistencia a tracción se ha basado en pruebas de tracción indirecta (ensayo brasileño) de acuerdo con la normativa ASTM-D3967 (9); en el caso del cálculo de la resistencia a flexión se han seguido las normativas UNI 9724/5 (10) y ASTM C99-87 (11). of weakness and very thin layers of silt deposits running parallel or subparallel to the bedding.

Today the quarries are located within the confines of a natural park where excavation is severely restricted by conservation law.

\section{MATERIALS AND METHODS}

Crinoid and micritic limestone blocks from the "Los Órganos 2" and "La Sombra" quarries located at Atarfe, Granada, on the slopes of Sierra Elvira, were used in this survey. A mineralogical and textural study of the samples was conducted with powder X-Ray Diffraction (XRD) and polarized optical microscopy. The instruments used were a Philips PW1710 diffractometer equipped with an automatic slit system and an Olympus BX-60 petrographic microscope.

Limestone colour was established with a HunterLab MiniScan $4000 S$ spectrophotometer fitted with a tungsten lamp light source. Tristimulus $(X, Y, Z)$, brightness $\left(L^{*}\right)$, chromaticity $\left(a^{*}, b^{*}\right)$, colour $\left(C^{*}\right)$ and hue angle $\left(H^{*}\right)$ values were obtained.

Limestone porosity was measured via both mercury intrusion porosimetry (MIP), using a Micromeritics 9410 AutoPore III system, and water resistance tests consisting in natural absorption (7) and desorption (8) in $5 \mathrm{~cm}$ cubic specimens.

The longitudinal elastic wave velocity, VP, was calculated from ultrasound measurements taken with a Controls model E46/3 54-kHz transducer ultrasonic pulse generator.

Finally, mechanical tests were conducted to determine stone resistance to (longitudinal and transversal) strain and compressive, tensile and bending strength. Strain was measured on $7 \times 7 \times 21 \mathrm{~cm}$ specimens using a Hottinger HBM 20/120 electric strain gauge. Seven-cm cubic specimens were subjected to dry uniaxial compression testing on a Metro Com MI 30 testing machine with a load capacity of $3 \times 10^{5} \mathrm{~kg}$. The test was carried out in dry conditions on $7 \mathrm{~cm}$-edge cubic specimens. Tensile strength was found via the indirect splitting tensile test (Brazilian test) to standard ASTM-D3967(9); bending strength was found as described in standards UNI 9724/5 (10) and ASTM C99-87 (11). 


\section{RESULTADOS Y DISCUSIÓN}

\subsection{Características petrográficas}

Caliza de crinoides. La principal característica macroscópica es la presencia de una estructura laminada a gran escala, así como una granulometría y composición variables. La piedra de Sierra Elvira se compone de granos y cemento de carbonato cálcico; se originó a partir de aportes de cieno o barro calizo en mares o lagos poco profundos, conteniendo además restos de fósiles y algas. La microestructura presenta elevada densidad, careciendo de una porosidad apreciable con microscopía óptica (12).

El color, desde el punto de vista estético varía de un verde grisáceo a gris con algunas bandas de calcita muy finas y venas de estilolitos de color marrón, amarillo o verde, subparalelos a la estratificación, que no influyen mucho en el cromatismo, especialmente cuando la roca está pulida. Su color predominantemente gris confiere a las obras arquitectónicas y elementos ornamentales un tono apagado y frío.

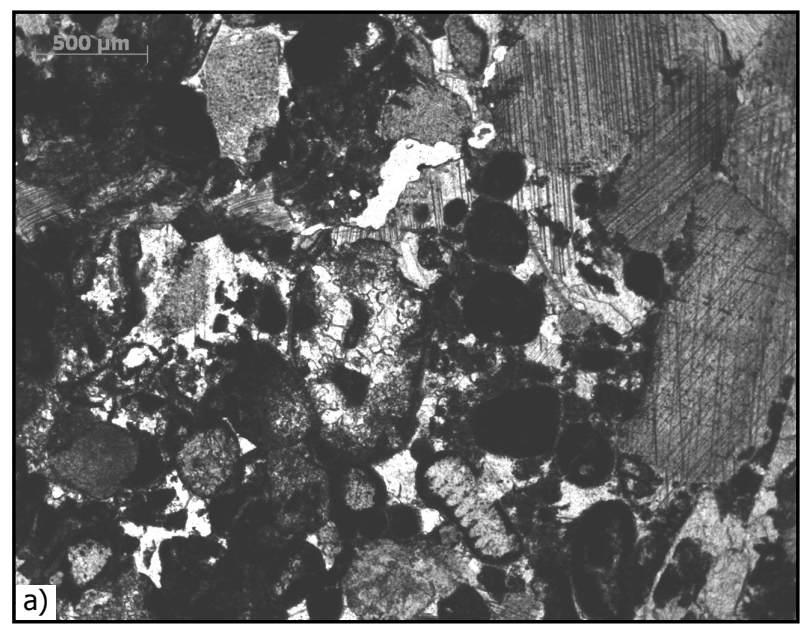

\section{RESULTS AND DISCUSSION}

\subsection{Petrographic characteristics}

Crinoid limestone. The most prominent macroscopic characteristics of this stone are its large-scale laminated structure and variable composition and particle size distribution. Sierra Elvira stone is composed of calcium carbonate grains and cement and is the result of the deposition of fossil- and alga-carrying calcareous mud or slime in shallow seas or lakes. Its microstructure is very dense and no porosity can be detected under the optical microscope (12).

From an aesthetic point of view, the stone varies in colour from grey-green to grey with some very thin strips of calcite and brown, yellow or green stylolite veins running subparallel to the beds whose impact on rock coloration is minimal, especially after polishing. This predominantly grey stone lends a cold, lustreless tone to buildings and ornamental elements.

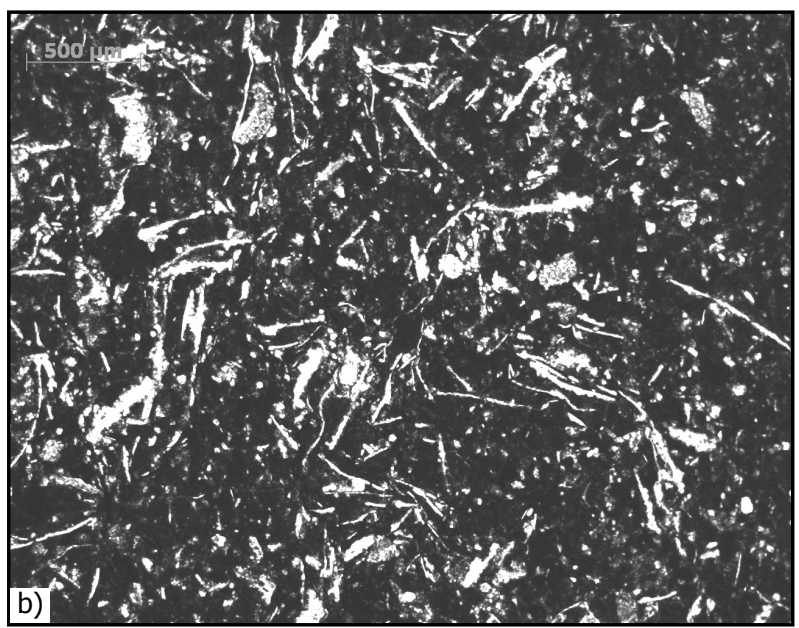

Figura 4. Imágenes al microscopio óptico de la caliza de crinoides (a) y micrítica (b).

Figure 4. Optical microscopic images of crinoid (a) and micritic (b) limestones.

Su estudio mediante microscopía óptica y DRX permite señalar que mineralógicamente presenta un dominio casi absoluto de calcita (Figura 4a); en algunos casos se encuentran también cristales de dolomita, así como algunos granos muy escasos de glauconitas, cuarzos, óxidos e hidróxidos de Fe y otros, siempre como fases minerales que rellenan los estilolitos (13).

Caliza micrítica. Esta variedad presenta granulometría lutítica. Macroscópicamente parece un wackstone, a veces con acumulaciones compactas de lamelibranquios pelágicos, mientras la observación microscópica revela aspecto de packstone y, en otros casos, una facies de transición.
The optical microscopy and $X R D$ results revealed that calcite is the majority mineral (Figure 4a), although in some cases the stylolite cavities contain dolomite crystals and occasional glauconite, quartz, Fe oxide or hydroxide grains or other minerals (13).

Micritic limestone. This variety of rock has a lutitic particle size distribution. To the naked eye it looks like wacke, on occasion exhibiting compact clusters of pelagic bivalves, while under the microscope it resembles packstone or in some cases a transition facies. 
El color en muestras de mano, es generalmente marrón bronce, si bien puede adquirir puntualmente tonos rosados. Su composición mineralógica es exclusivamente calcítica (Figura 4b).

\subsection{Propiedades Físicas}

Propiedades Físicas Elementales. Los valores obtenidos de colorimetría, media de 120 medidas de cada variedad de caliza, se presentan en la Tabla 1.

Los efectos del pulimento comportan una variación del color de $\Delta \mathrm{E}^{*}=15,7$ y $\Delta \mathrm{E}^{*}=14,1$ respectivamente para la caliza con crinoides y para la micrítica. Asimismo, produce una disminución del brillo, mientras no aumenta la saturación. Este comportamiento es parecido al de los mármoles y otros tipos de rocas ornamentales de carácter marcadamente cristalino (14); sin embargo, difieren apreciablemente de los de piedras ornamentales que presentan parámetros cromáticos altos (15).
The colour is generally bronze-brown, although a pinkish hue is not uncommon. Calcite is its sole mineral constituent (Figure 4b).

\subsection{Physical Properties}

Elementary physical properties. The colorimetric values obtained - the mean of 120 measurements for each variety of limestone - are given in Table 1.

Polishing brings about a colour variation of $\Delta \mathrm{E}^{*}=15.7$ and $\Delta \mathrm{E}^{*}=14.1$ for crinoid and micritic limestone, respectively. It also lowers brightness without raising saturation levels. Similar behaviour in this regard has been reported for marble and other eminently crystalline ornamental rocks (9), whereas high chromaticity decorative stone exhibits widely different patterns (15).

Tabla 1 / Table 1

Valores triestimulo $(X, Y, Z)$, coordenadas cromáticas $(x, y)$, de luminosidad $\left(L^{*}\right)$, cromaticidad $\left(a^{*}, b^{*}\right)$, color $\left(\mathrm{C}^{*}\right)$ y ángulo de matiz $\left(\mathrm{H}^{\circ}\right)$ de las dos variedades de calizas.

Tristimulus values $(X, Y, Z)$, chromatic coordinates $(x, y)$, brightness $\left(L^{*}\right)$, chromaticity $\left(a^{*}, b^{*}\right)$, colour $\left(C^{*}\right)$ and hue angle $\left(H^{\circ}\right)$ for two varieties of limestone.

\begin{tabular}{|l|c|c|c|c|c|c|c|c|c|c|}
\cline { 2 - 10 } \multicolumn{1}{c|}{} & $\mathbf{X}$ & $\mathbf{Y}$ & $\mathbf{Z}$ & $\mathbf{X}$ & $\mathbf{y}$ & L* & a* & b* & C* & H* \\
\hline Caliza crinoides / Crinoid limestone & 18.30 & 18.50 & 16.80 & 0.34 & 0.35 & 49.50 & 0.30 & 7.90 & 7.90 & $88^{\circ}$ \\
\hline Caliza micrítica / Micritic limestone & 15.70 & 15.60 & 12.30 & 0.36 & 0.36 & 46.00 & 3.10 & 17.00 & 17.30 & $80^{\circ}$ \\
\hline
\end{tabular}

Los valores de luminosidad ( $L^{*}$, Tabla 1 ) se sitúan en ambos litotipos por debajo del $50 \%$, siendo ligeramente más oscura la variedad micrítica. Las diferencias entre los dos tipos de calizas aumentan si consideramos la cromaticidad (parámetros a* y b*, Tabla 1) dado que son más altos en el caso de la caliza micrítica.

Otros parámetros técnicos establecidos son los que se presentan en la Tabla 2. Todos los valores calculados, referidos a las dos variedades, son muy similares. Notar como los valores de peso específico ( $\gamma_{r}$, Tabla 2$)$ son típicos de las rocas carbonatadas y muy cercanos al de la
Brightness ( $L^{*}$, Table 1) was under $50 \%$ in both varieties and slightly lower in the micritic stone. Greater differences between the two types of limestone were found when analyzed for chromaticity (parameters $a^{*}$ and $b^{*}$, Table $1)$, which was higher in the micritic variety.

The values of other technical parameters were also determined and, as shown in Table 2, found to be similar in the two stones. Specific weight ( $\gamma_{r}$, Table 2), for instance, was typical of values for carbonate rock, calcite in particular $\left(2.71 \mathrm{~g} / \mathrm{cm}^{3}\right)$. The pore index (e, Table 2$)$, was

Tabla 2 / Table 2

Parámetros físicos de las calizas de Sierra Elvira. $\gamma_{\mathrm{r}}=$ peso específico $\left(\mathrm{en} \mathrm{g} / \mathrm{cm}^{3}\right)$;

$\mathrm{C}=$ grado de compacidad; $\mathrm{n}=$ coeficiente de porosidad (en \%); e = índice de poros.

Physical properties of Sierra Elvira limestone $\gamma_{\mathrm{r}}=$ specific weight (in $\left.\mathrm{g} / \mathrm{cm}^{3}\right)$;

$C=$ degree of compactness; $n=$ porosity coefficient (in \%); $e=$ pore index.

\begin{tabular}{|l|c|c|c|c|}
\cline { 2 - 5 } \multicolumn{1}{c|}{} & $\gamma_{\mathbf{r}}$ & $\mathbf{c}$ & $\mathbf{n}$ & $\mathbf{e}$ \\
\hline Caliza crinoides / Crinoid limestone & 2.731 & 0.98 & 2 & 0.02 \\
\hline Caliza micrítica / Micritic limestone & 2.699 & 0.98 & 2 & 0.02 \\
\hline
\end{tabular}


calcita $\left(2,71 \mathrm{~g} / \mathrm{cm}^{3}\right)$. El índice de poros (e, Tabla 2 ) bastante bajo se debe únicamente a la presencia de fisuras secundarias en estos materiales.

Los datos de porosidad obtenidos mediante PIM oscilan entre $1,84 \%$ y $2,74 \%$ en el caso de las calizas con crinoides, mientras que los valores de las calizas micríticas son algo más elevados, alrededor de 3,40\%. Son unos parámetros de porosidad muy bajos, similares a los de una gran parte de rocas de textura cristalina, como son granitos y mármoles; aunque bastante inferiores a los de otras rocas calizas ornamentales como la Piedra de Borriol (16) o la caliza Gris Pulpis (17).

\subsection{Parámetros hídricos}

Estos parámetros permiten entender el comportamiento del material lapídeo respecto a los movimientos de los fluidos, principalmente el agua, y poder establecer una valoración bastante correcta de su futura alterabilidad. La absorción libre y secado de las calizas se han determinado pesando las muestras a intervalos regulares. La capacidad de absorber agua es muy baja, típica de rocas cristalinas muy compactas (Tabla 3). En la Figura 5 se puede apreciar cómo la variedad micrítica absorbe más agua que la caliza de crinoides. very low in these materials and their porosity due exclusively to the presence of secondary fissures.

The MIP findings ranged from $1.84 \%$ to $2.74 \%$ in crinoid limestone, but were somewhat higher in micritic limestone, at around $3.40 \%$. Such low porosity values are similar to the figures reported for many rocks with a crystalline texture such as granite and marble and substantially lower than in other varieties of ornamental limestone such as Piedra de Borriol (16) or Gris Pulpis (17)

\subsection{Water resistance}

Water resistance parameters provide insight into the behaviour of stone when exposed to the action of fluids, water in particular, and can be used to predict its propensity to decay in the future with some degree of certainty. Natural absorption and desorption were determined in the two varieties of limestone by weighing the specimens at regular intervals. Water absorbability was found to be very low, a characteristic typical of highly compact crystalline rocks (Table 3). Figure 5 shows that micritic limestone absorbed more water than the crinoid variety.

Tabla 3 / Table 3

Parámetros hídricos de las calizas de Sierra Elvira. $\mathrm{H} \%=$ contenido en humedad natural; $\mathrm{A} \%=$ coeficiente de absorción; $S \%=$ coeficiente de saturación; $D_{A}=$ densidad aparente $\left(e n g / \mathrm{cm}^{3}\right) ; D_{R}=$ densidad real (en $\left.\mathrm{g} / \mathrm{cm}^{3}\right) ; \mathrm{P}_{\mathrm{A}} \%$ = porosidad accesible al agua; $\mathrm{I}_{\mathrm{p}} \%=$ índice de porosidad calculada en función de $\mathrm{P}_{\mathrm{A}} \%$. Sierra Elvira limestone water resistance parameters. $H \%=$ natural humidity content; $A \%=$ absorption coefficient; $S \%=$ saturation coefficient; $D_{A}=$ apparent density (in $\left.\mathrm{g} / \mathrm{cm}^{3}\right) ; D_{R}=$ real density (in $\mathrm{g} / \mathrm{cm}^{3}$ ); $P_{A} \%=$ open porosity; $I_{p} \%=$ porosity level calculated from $P_{A} \%$.

\begin{tabular}{|l|c|c|c|c|c|c|c|}
\cline { 2 - 7 } \multicolumn{1}{c|}{} & $\mathbf{H}$ & $\mathbf{A}$ & $\mathbf{S}$ & $\mathbf{D}_{\mathbf{A}}$ & $\mathbf{D}_{\mathbf{R}}$ & $\mathbf{P}_{\mathbf{A}}$ & $\mathbf{I}_{\mathbf{p}}$ \\
\hline Caliza crinoides / Crinoid limestone & 0.024 & 0.09 & 0.26 & 2.71 & 2.71 & 0.20 & 1.002 \\
\hline Caliza micrítica / Micritic limestone & 0.035 & 0.14 & 0.38 & 2.69 & 2.70 & 0.37 & 1.004 \\
\hline
\end{tabular}

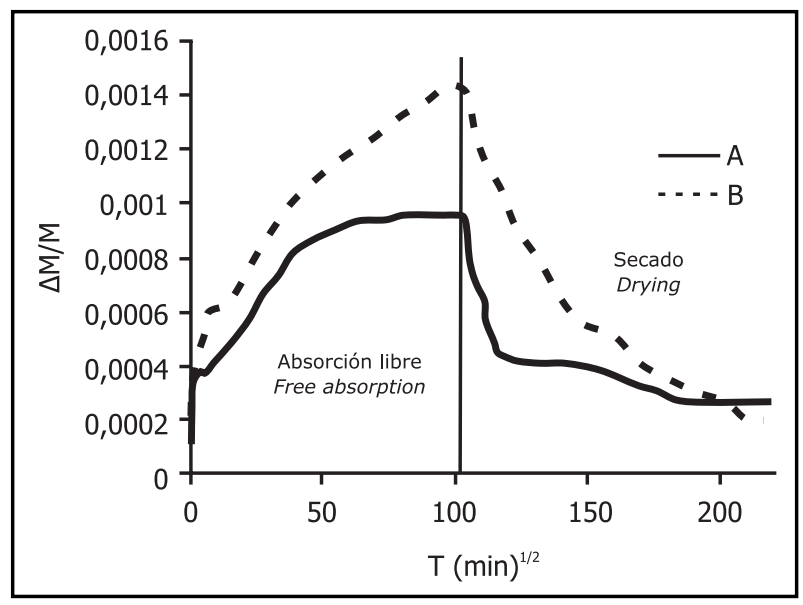

Figura 5. Curvas de absorción libre de agua y secado en las calizas de crinoides (A) y micrítica (B). Figure 5. Natural water absorption and desorption curves for crinoid (A) and micritic (B) limestone. 


\subsection{Propiedades Dinámicas. Velocidad de las ondas elásticas longitudinales}

Mediante la técnica de propagación de ultrasonidos se han determinado los valores de los coeficientes de anisotropías absoluta y relativa de estas rocas (18). Los valores de anisotropía obtenidos (Tabla 4) son bastante inferiores a los de otras rocas ornamentales; así p.e. los mármoles Blanco y Amarillo Triana de Macael exceden el 10,5\% (19) o los de las calcarenitas de Santa Pudia, ampliamente usada en el Patrimonio Arquitectónico de Granada, que son superiores al 5,5\% $(20,21)$.

\subsection{Anisotropy. Longitudinal elastic wave velocity}

Ultrasound propagation (18) was used to find absolute and relative rock anisotropy coefficients. The anisotropy values obtained (Table 4) were considerably lower than reported for other ornamental rocks, such as the 10.5\% reported for white and yellow Triana Macael marble, or the $5.5 \%$ found in the calcarenite quarried at Santa Pudia, also widely used to build Granada's architectural heritage $(20,21)$.

Tabla 4 / Table 4

$\mathrm{V}_{\mathrm{p} 1}, \mathrm{~V}_{\mathrm{p} 2}$ y $\mathrm{V}_{\mathrm{p} 3}$ representan las velocidades de transmisión de ondas ultrasónicas (en $\mathrm{m} / \mathrm{s}$ ) en las direcciones perpendiculares y paralelas $\left(\mathrm{V}_{\mathrm{p} 2}\right.$ y $\left.\mathrm{V}_{\mathrm{p} 3}\right)$ al plano de estratificación. $\Delta \mathrm{M}$ y $\Delta \mathrm{m}$ son los índices de anisotropía total y relativa (en \%) calculados en función de los datos anteriores.

Ultrasonic wave transmission velocities (in $\mathrm{m} / \mathrm{s}$ ) in directions perpendicular and parallel $\left(V_{p 2}\right.$ and $\left.V_{p 3}\right)$ to the bedding plane and respective total $(\Delta M)$ and relative $(\Delta m)$ anisotropy values (in \%)

\begin{tabular}{|l|c|c|c|c|c|}
\cline { 2 - 5 } \multicolumn{1}{c|}{} & $\mathbf{V}_{\mathbf{p} \mathbf{1}}$ & $\mathbf{V}_{\mathbf{p 2}}$ & $\mathbf{V}_{\mathbf{p} \mathbf{3}}$ & $\mathbf{\Delta M}$ & $\Delta \mathbf{m}$ \\
\hline Caliza crinoides / Crinoid limestone & $5779 \pm 558$ & $6013 \pm 340$ & $5946 \pm 388$ & $3.6 \pm 5.1$ & $1.3 \pm 1.5$ \\
\hline Caliza micrítica / Micritic limestone & $5696 \pm 293$ & $5884 \pm 256$ & $5843 \pm 372$ & $3.5 \pm 2.3$ & $2.2 \pm 1.1$ \\
\hline
\end{tabular}

Por otra parte, los valores de las $\mathrm{V}_{\mathrm{p}}$ (ligeramente más bajos los de la caliza micrítica) nos indican la alta calidad dinámica de estas piedras ornamentales. Si comparamos estos datos con los encontrados en el mármol blanco de Macael (19) o en rocas metamórficas foliadas (22) se observa que son muy similares.

\subsection{Comportamiento Elástico}

Ambas calizas se caracterizan por un comportamiento típicamente frágil. Las curvas (Figura 6) permiten establecer el módulo de Young, que oscila entre $76092 \mathrm{MPa}$ y $80531 \mathrm{MPa}$, en función de la orientación de la probeta respecto al plano de estratificación y el correspondiente coeficiente de Poisson ( $v_{\text {sec }} 50$ ) entre 0,29 y 0,34 (13). Los valores del módulo de Young obtenidos son típicos de calizas resistentes.

\subsection{Propiedades Mecánicas}

La caliza de crinoides presenta valores de resistencia altos; la mayor resistencia coincide con la dirección en que la carga aplicada es perpendicular al plano de estratificación. Las diferencias entre las tres direcciones ortogonales están en el rango del centenar de $\mathrm{kg} / \mathrm{cm}^{2}$; se advierte una buena correlación con el bajo índice de anisotropía total $\Delta \mathrm{M}$ (Tabla 4) comentado anteriormente en relación a los datos de $V_{p}$.

Los valores de resistencia a flexión son: caliza de crinoides $189 \pm 24 \mathrm{~kg} / \mathrm{cm}^{2}$, mientras que en el caso de la cali-
The relatively high $v_{p}$ values (slightly lower for micritic limestone) found, which were similar to the figures for white Macael marble (19) and foliated metamorphic rocks (22), are indicative of the low anisotropy of these ornamental stones.

\subsection{Elastic behaviour}

Both types of limestone proved to be fragile. Their Young moduli, determined from the curves in Figure 6, were shown to depend on the orientation of the specimen with respect to the bedding plane. The values found, ranging from $76092 \mathrm{MPa}$ to $80531 \mathrm{MPa}$, were typical of resistant limestone. The respective Poisson coefficients $\left(v_{\mathrm{sec}} 50\right)$, in turn, varied from 0.29 to 0.34 (13).

\subsection{Mechanical properties}

Crinoid limestone exhibited high strength values, particularly when the load was applied perpendicularly to the bedding plane. The values for the three orthogonal directions varied by only about $100 \mathrm{~kg} / \mathrm{cm}^{2}$, corroborating the low total anisotropy value $\Delta M$ (Table 4) obtained above.

The bending strength values - $189 \pm 24 \mathrm{~kg} / \mathrm{cm}^{2}$ for crinoid limestone and a slightly lower $127 \pm 33 \mathrm{~kg} / \mathrm{cm}^{2}$ for 


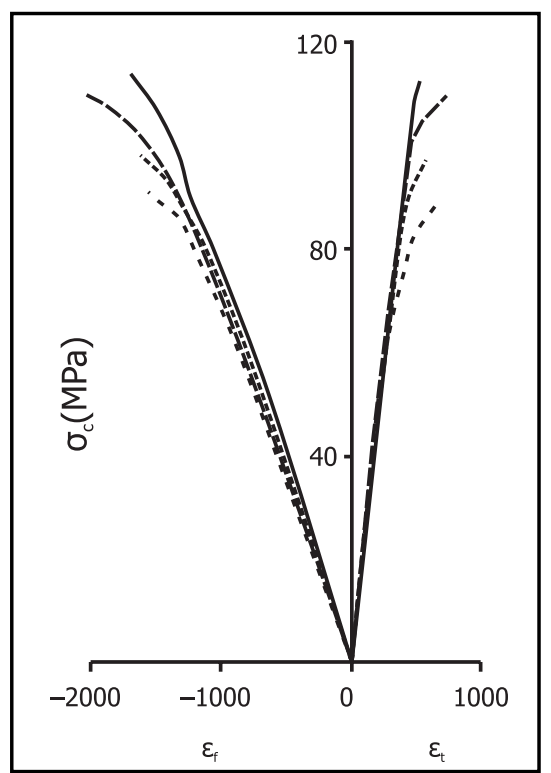

Figura 6. Curvas esfuerzo ( $\sigma_{\mathrm{c}}$ en MPa) - deformación $\left(\varepsilon_{\mathrm{f}}\right.$ y $\left.\varepsilon_{\mathrm{t}}\right)$ de muestras de caliza de crinoides de Sierra Elvira.

Figure 6. Stress-strain curves for Sierra Elvira crinoid limestone.

za micrítica es algo más bajo $127 \pm 33 \mathrm{~kg} / \mathrm{cm}^{2}$; datos parecidos a los de otras rocas de construcción de características composicionales y texturales similares (17).

Los datos de resistencia a tracción de la caliza de crinoides es $92 \pm 12,1 \mathrm{~kg} / \mathrm{cm}^{2}$, aunque en secciones de carga paralelas a la laminación es algo menor: $84 \pm 13,5$ $\mathrm{kg} / \mathrm{cm}^{2}$; mientras que los relativos a la caliza micrítica son: $86 \pm 15,5 \mathrm{~kg} / \mathrm{cm}^{2}$, en secciones paralelas se ha obtenido $71 \pm 19 \mathrm{~kg} / \mathrm{cm}^{2}$.

Indicar que los datos de la Tabla 5 (que representan la media de 16 medidas) decaen alrededor del $25 \%$ en probetas que hayan sido sometidas a ensayos de hielo-deshielo de acuerdo con la normativa RILEM (23). micritic limestone - were in line with the figures reported for other building stones with similar textural and compositional characteristics (17).

Tensile strength in crinoid limestone was $92 \pm 12.1$ $\mathrm{kg} / \mathrm{cm}^{2}$, although in lamination-parallel load sections it dropped to $84 \pm 13.5 \mathrm{~kg} / \mathrm{cm}^{2}$. The respective values for micritic limestone were $86 \pm 15.5 \mathrm{~kg} / \mathrm{cm}^{2}$ and $71 \pm 19$ $\mathrm{kg} / \mathrm{cm}^{2}$ in parallel sections.

Strength in specimens that had been subjected to freezethaw cycles as per the RILEM standard (23), in turn, was almost 25\% lower (see Table 5, which gives the average of 16 measurements).

Tabla 5 / Table 5

Valores de la resistencia a compresión uniaxial establecidos a partir de material seco. Uniaxial compressive strength of dry specimens.

\begin{tabular}{|l|c|c|c|}
\cline { 2 - 4 } \multicolumn{1}{c|}{} & $\begin{array}{c}\text { Carga // laminación } \\
\text { plano } a-b / \text { Load // } \\
\text { lamination on plane } a-b\end{array}$ & $\begin{array}{c}\text { Carga // laminación } \\
\text { plano } a-c \text { / Load // } \\
\text { lamination on plane } a-c\end{array}$ & $\begin{array}{c}\text { Carga // laminación } \\
\text { plano } b-c / \text { Load // } \\
\text { lamination on plane } b-c\end{array}$ \\
\hline Caliza crinoides / Crinoid limestone & $\begin{array}{c}1432 \pm 340 \mathrm{~kg} / \mathrm{cm}^{2} \\
140.3 \pm 33.3 \mathrm{MPa}\end{array}$ & $\begin{array}{c}1309 \pm 200 \mathrm{~kg} / \mathrm{cm}^{2} \\
128.3 \pm 19.6 \mathrm{Mpa}\end{array}$ & $\begin{array}{c}1400 \pm 236 \mathrm{~kg} / \mathrm{cm}^{2} \\
137.2 \pm 23.1 \mathrm{MPa}\end{array}$ \\
\hline \multirow{2}{*}{ Caliza micrítica / Micritic limestone } & $1564 \pm 225 \mathrm{~kg} / \mathrm{cm}^{2}$ & $1479 \pm 313 \mathrm{~kg} / \mathrm{cm}^{2}$ & $1692 \pm 192 \mathrm{~kg} / \mathrm{cm}^{2}$ \\
& $153.3 \pm 22.0 \mathrm{MPa}$ & $144.9 \pm 30.7 \mathrm{MPa}$ & $165.8 \pm 18.8 \mathrm{MPa}$ \\
\hline
\end{tabular}

\section{ALTERACIÓN Y CONSERVACIÓN DE LA PIEDRA DE SIERRA ELVIRA}

La degradación, sobre todo de carácter químico, de esta piedra ornamental es de escasa intensidad y muy lenta;

\section{ALTERATION AND CONSERVATION OF SIE- RRA ELVIRA STONE}

Decay, in particular chemical decay, is very slow and scantly intense in this stone, owing to its physical and 
ello se debe a sus parámetros físico-mecánicos, esencialmente sus índices de porosidad eficaz y su comportamiento frente a la captación de fluidos y gases, así como su circulación por el interior de la piedra, ya que el limitado tamaño de microporo, su escasa conectividad, y la exigua permeabilidad la restringen significativamente. Su comportamiento frente a la contaminación también es aceptable debido a estas mismas causas.

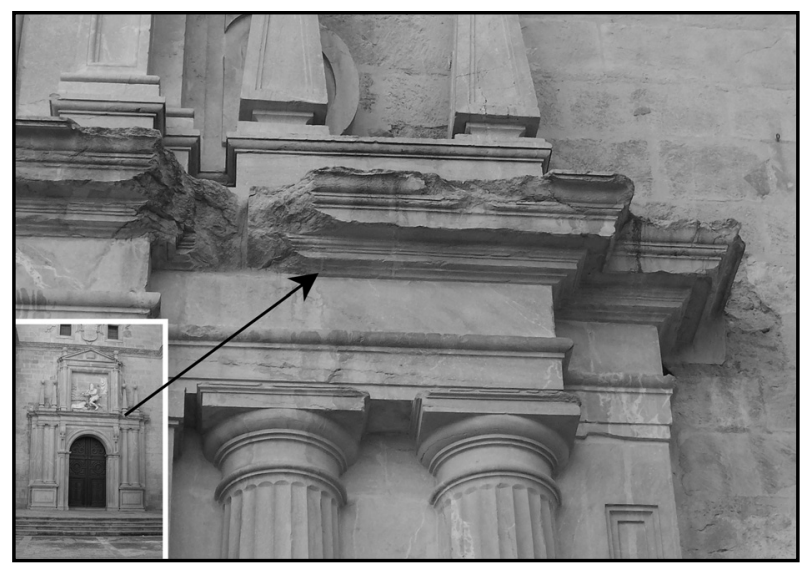

Figura 7. Daños por pérdidas de material en una moldura de la portada del Monasterio de San Jerónimo (Granada).

Figure 7. Partial destruction of a portal moulding, on St Jerome Monastery, Granada.

Su durabilidad viene motivada, además, por sus excelentes propiedades mecánicas, dinámicas y el bajo coeficiente de anisotropía estructural-textural; este último aspecto se refleja en los escasos daños generados por esfuerzos mecánicos debidos a dilataciones y contracciones asociadas a variaciones térmicas acusadas, fenómeno climático usual en Granada y que, sin embargo, sí representa un factor determinante en el deterioro de otros tipos de rocas ornamentales, como sucede con los mármoles (24-26).

Los daños más frecuentes son los originados por defectos estructurales de la construcción, los ocasionados por impactos, los debidos a las discontinuidades y heterogeneidades intrínsecas, como son los estilolitos, y cuando esta piedra se expone a procesos de contaminación ambiental importante (Figuras 7 y 8 ). Las morfologías de deterioro más llamativas son las pérdidas de material, las rupturas y las pátinas o acumulaciones de suciedad; con menor frecuencia la decohesión granular.

Las fases de restauración/conservación más habituales a emprender en este tipo de roca serán la limpieza y, en su caso, la reintegración, en menor medida la protección y consolidación. Estos dos últimos procedimientos vienen muy condicionados por la escasa eficacia que logran los productos y metodologías de aplicación más usuales en intervenciones en piedra ornamental, ya que la penetra- mechanical properties. Its effective porosity, which determines its permeability to liquids and gases, is particularly significant in this respect. Fluids are barely able to circulate inside the stone because the small size and poor connectivity of its micropores make it virtually impermeable. These same attributes provide adequate protection from pollution.

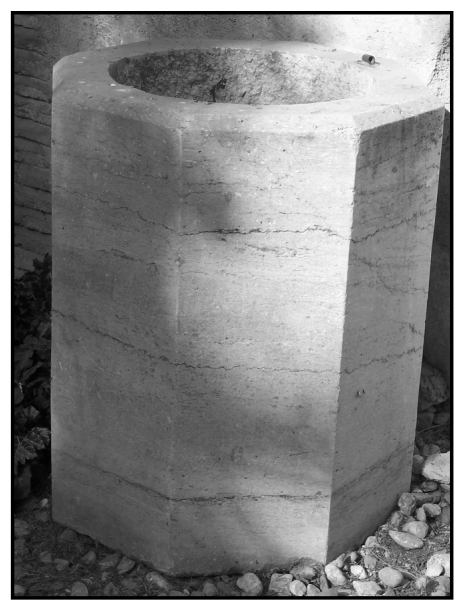

Figura 8. Discontinuidades en la caliza debidas a la presencia de estilolitos.

Figure 8. Stylolite discontinuities in limestone.

Its durability is also the result of its excellent mechanical properties and low textural and structural anisotropy coefficient. Proof of this is to be found in the scant damage caused by the expansion and contraction-induced mechanical stress associated with the extreme variations in temperature that characterize Granada. Such variations are a determinant in the decay of other kinds of ornamental rock such as marble (24-26).

The most frequent causes of decay are structural flaws, the impact of objects of whatever nature, intrinsic discontinuities and heterogeneities such as stylolites and exposure to severe environmental pollution (Figures 7 and 8). Decay adopts many different forms including the partial destruction of elements, fracture and accumulated dirt. Crumbling also occurs, although less frequently.

Restoration and conservation work on this type of rock normally consists in cleaning and repair as necessary, and to a lesser extent protection and consolidation. The latter two measures have proven to be largely ineffective in ornamental stone, however, for penetration and 
ción o fijación superficial en esta roca es casi nula debido a su espectro porosimétrico.

\section{CONCLUSIONES}

La caracterización petrofísica-mecánica pone de manifiesto la notable calidad técnica de esta piedra natural de construcción, que puede ser penalizada: por la geometría, extensión y densidad de los estilolitos, la posible presencia en su relleno de fases minerales como glauconitas y óxidos e hidróxidos de $\mathrm{Fe}$, o bien la forma de su puesta en obra. Se recomienda, por tanto, la selección de bloques con la mínima densidad de estilolitos y sin fisuras.

El hecho de la ubicación de la piedra de Sierra Elvira en zonas muy específicas de los edificios ya nos indica que tiene unas características mecánicas determinadas; sus elevados valores de resistencia a compresión, al impacto y al desgaste la convierten en un material idóneo para la elaboración de columnas, pedestales, escaleras, en forma de losas para solería y zócalos, o también en piezas regulares en forma de adoquines o para conformar bordillos en calles. Los bajos índices de anisotropía mecánica permiten, por otra parte, su modelado mediante cincelado.

La resistencia mecánica es alta incluso en la variedad micrítica a pesar de las abundantes discontinuidades que presenta, especialmente, fracturas más o menos latentes, venas calcíticas subparalelas al plano de estratificación, estilolitos y alteración superficial fácil.

Este material de construcción resulta ser una caliza de una notable durabilidad en las condiciones climáticas de régimen continental de la ciudad de Granada. También, frente a la gran mayoría de los factores extrínsecos de deterioro de las rocas ornamentales, como el agua, agentes biológicos, presencia de sales o contaminación atmosférica.

A causa de su reducida porosidad y elevada razón micro a macroporosidad, las únicas etapas de intervención eficaces son las de limpieza de pátinas, costras, depósitos, y de reintegración. La durabilidad no mejora mediante la aplicación de productos de protección; sí la incrementa el pulimento habitual de las fábricas, que no suele modificar significativamente su cromaticidad.

Los usos ornamentales y estructurales de la caliza de Sierra Elvira parecen satisfacer todos los requerimientos estéticos y de comportamiento mecánico. Su utilización es recomendable tanto en edificios históricos como para rehabilitación arquitectónica o en nueva construcción. superficial bonding are almost nil in such rock, due to its scant porosity.

\section{CONCLUSIONS}

This description of the petrophysical and mechanical properties of Sierra Elvira stone explains why it is such an excellent natural building material. Its performance may be adversely affected by poor construction techniques, as well as by stylolite geometry, length and density and the possible presence of mineral phases such as glauconites or compounds such as Fe oxides and hydroxides in these cavities. Consequently, only blocks with a minimum stylolite density and no fissures should be selected for building.

The fact that Sierra Elvira stone was used in very specific areas of buildings is an indication that it was known to have certain mechanical properties. Its high compressive strength and impact- and wear-resistance made it an ideal material for columns, pedestals, staircases, flooring and baseboards, as well as for pavement cobbles or curbing. Thanks to its low mechanical anisotropy, it can be chiselled to different shapes.

Mechanical strength is high even in the micritic variety, despite its many discontinuities, including visible or latent fractures, calcitic veins subparallel to the bedding plane, stylolites and low resistance to superficial decay.

This building material has been shown to be very durable, even in the city of Granada's typically continental climate. It is also resistant to most of the extrinsic factors that damage ornamental rocks, such as water, biological agents, salt and air pollution.

Due to its extremely low porosity levels and high micro/macro porosity ratio, the only effective conservation techniques are the removal of patinas, black crusts or other deposits and the repair of damaged stones. Protective materials do not increase stone durability, which is nonetheless enhanced by standard factory polishing, a practice that has no significant effect on stone colour.

Inasmuch as Sierra Elvira limestone seems to meet all the necessary aesthetic and mechanical requirements for ornamental and structural use, it is recommended for both the restoration of historic buildings and new construction. 


\section{AGRADECIMIENTOS}

Los autores agradecen la financiación del Proyecto de investigación MEC MAT2004-6804 y el Grupo de Investigación RNM179 de la Junta de Andalucía. Agradecen además a Nigel Walkington la traducción del manuscrito.

\section{ACKNOWLEDGEMENTS}

The present study was funded by Spanish Ministry of Education and Science Research Project MEC MAT20046804 and Andalusian Regional Government Research Team RNM179. The authors wish to thank Nigel Walkington for translating the manuscript.

\section{BIBLIOGRAFÍA / BIBLIOGRAPHY}

(1) IGME: "Mármoles españoles", Ministerio de Industria y Energía, Madrid, (1990).

(2) Cantó, A. M.: "Avances sobre la explotación del mármol en la España romana", Archivo Español de Arqueología, Vol. 51, (1978), pp. $165-188$.

(3) Cisneros Cunchillos, M.: "Mármoles hispanos: su empleo en la España romana", Monografía, Universidad de Zaragoza, (1988).

(4) Arance, J., Medina, L. J.: "La piedra de Sierra Elvira como material de construcción tradicional en Granada", Escuela Universitaria de Arquitectura Técnica, Universidad de Granada, (1998).

(5) Linares, A., Rivas, P.: "El Lías Medio en la parte sur de la Zona Subbetica (Sierra Elvira, Illora y Iznalloz, Provincia de Granada)", Cuadernos de Geología, Vol. 4 (1973), pp. 141-154.

(6) Dabrio, C. J., Polo, D.: "Interpretación sedimentaria de las calizas de crinoides del Carixiense Subbético", Mediterranea, Vol. 4 (1985), 55-75.

(7) NORMAL 7/81: "Assorbimento dell'acqua per immersione totale. Capacità di imbibizione", CNR-ICR, Roma, (1981).

(8) NORMAL 29/88: "Misura dell'indice di asciugamento (drying index)", CNR-ICR, Roma, (1988).

(9) ASTM D 3967-86: "Splitting Tensile Strength of Intact Stone Core Specimens", American Society of Testing and Materials, Philadelphia, Pennsylvania, (1986).

(10) UNI 9724/5: "Materiali lapidei. Determinazione Della resistenza a flessione", CNR-ICR, Roma, (1990).

(11) ASTM C 99-87: "Standard test methods for determining the modulus of rupture of dimension stone", American Society of Testing and Materials, Philadelphia, Pennsylvania, (2000).

(12) García Hernández, M, Rivas, P., Vera, J. A.: "El Carixiense en la Zona Subbética", Cuadernos de Geología, Vol. 10, (1979), pp. (375-382). (13) Garibaldi, V.: "Studio petrografico e fisico-meccanico del calcare a crinoidi utilizzato nell' architettura di Granada (Andalucía, Spagna)", Tesi Sperimentale di Laurea, Università di Pavia (Italia), (1999).

(14) Zezza, U., Massa, V., Cuzzolin, M.: "Non destructive spectrophotometric colour testing on granites from "Lago Maggiore (Italy)", Actas IV Congreso Internacional de Rehabilitación del Patrimonio Arquitectónico. CICOP, La Habana, (1998), pp. (527-529).

(15) Benavente, E., Martínez Verdú, F., Bernabeu, A., Viqueira, V., Fort, R., García del Cura, M.A., Illueca, C., Ordóñez, S.: "Influence of surface roughness on color changes in building stones", Color Research and Application, Vol. 28, 2003, pp. (343-351).

(16) Ovejero, M., Queralt, I., de la Fuente, C.: "Caracterización petrográfica y comportamiento hídrico de diferentes litotipos de la Piedra de Borriol (Castellón)", Mater. Construcc., Vol. 55, (2005), pp. 41-54.

(17) García del Cura, M. A., Benavente, D., Bernabeu, A., Fort, R., La Iglesia, A., Ordóñez, S.: "Las calizas microcristalinas como material de construcción: el caso del Gris Pulpis", Mater. Construcc., Vol., 55, (2005), pp. 5-23.

(18) Guydader, J., Denis, A.: "Propagation des ondes dans les roches anisotropes sous contrainte évaluation de la qualité des schistes ardoisiers", Bulletin Engineering Geology, Vol. 33, (1986), pp. 49-55.

(19) Sebastián Pardo, E., Rodríguez-Navarro, C., Cultrone, G., Urosevic, M., Rodríguez-Navarro, A.: "Caracterización de la anisotropía textural de mármoles comerciales", Macla, Vol. 6, (2006), 453-456.

(20) Rodríguez-Navarro, C., Sebastián Pardo, E., Zezza, U., De La Torre, M.J., Cardell, C.: "Caracterización mineralógica y petrofísica de los materiales biocalcareníticos utilizados en la construcción de monumentos históricos de Granada", Boletín de la Sociedad Española de Mineralogía, Vol. 14, (1991), pp. 25-26.

(21) Luque, A., Cultrone, G., Sebastián, E., Cazalla, O.: "Evaluación de la eficacia de tratamientos en el incremento de la durabilidad de una calcarenita bioclástica (Granada, España)", Mater. Construcc., en prensa.

(22) Song, I., Suh, M., Woo Y. K., Hao, T.: "Determination of the elastic modulus set of foliated rocks from ultrasonic velocity measurements", Engineering Geology, Vol. 72, (2004), pp. 293-308.

(23) RILEM: "Recommended tests to measure the deterioration of stone and to assess the effectiveness of treatment methods", Commission 25-PEM: Protection et Erosion des Monuments, (1980), 175-253.

(24) Zezza, U., Sebastián Pardo, E.M.: "El mármol de Macael en los monumentos históricos de Granada (España)". Actas I Cong. Int. Rehabilitación del Patrimonio Arquitectónico, Islas Canarias, Vol. 1, (1992), pp.153-162.

(25) Zezza, U., Sebastián Pardo, E.M.: "Conservation of the Lions Court at the Alhambra of Granada", IABSE Symposium Rome: Structural Preservation of the Architectural Heritage, Vol. 70, (1993), pp. 725-726.

(26) Sáez Pérez, M. P. "Estudio de elementos arquitectónicos y composición de materiales del Patio de los Leones. Interacciones en sus causas de deterioro", Tesis Doctoral, Universidad de Granada (2003). 